Service social

\title{
L'approche structurelle en travail social : implications pratiques d'une approche intégrée conflictuelle
}

\section{Maurice Moreau}

Volume 36, numéro 2-3, 1987

Approches intégrées

URI : https://id.erudit.org/iderudit/706361ar

DOI : https://doi.org/10.7202/706361ar

Aller au sommaire du numéro

Éditeur(s)

École de service social de l'Université Laval

ISSN

1708-1734 (numérique)

Découvrir la revue

Citer cet article

Moreau, M. (1987). L'approche structurelle en travail social : implications pratiques d'une approche intégrée conflictuelle. Service social, 36(2-3), 227-247. https://doi.org/10.7202/706361ar
Résumé de l'article

Cet article fait le point sur l'approche structurelle, mode d'intervention qui s'est inspiré des tendances les plus radicales en service social.

S'appuyant sur cinq pratiques interreliées, l'auteur décrit l'approche structurelle, en montre les implications concrètes, y discute les techniques d'intervention les plus fréquemment utilisées et identifie certains dilemmes qu'elle soulève. 


\section{L'approche structurelle en travail social : implications pratiques d'une approche intégrée conflictuelle ${ }^{1}$}

\section{Maurice Moreau}

L'approche structurelle (Moreau, 1979, 1982) à la fois intégrée et conflictuelle, inspirée des perspectives et des pratiques radicales en service social (Léonard, 1975; Gulper, 1975, 1980) a presque quinze ans d'existence.

L'approche structurelle faisant appel à cinq pratiques interreliées, nous préciserons les implications concrètes de chacune d'elles et nous identifierons les techniques d'intervention auxquelles elles font appel. En conclusion, certains dilemmes et contradictions que soulève l'approche seront discutés.

Ces cinq pratiques (Bolger et al., 1981; Simplin, 1983; Statham, 1978 ; Stephen et Black, 1985 ; Walker et Beaumont, 1981) sont la matérialisation et la collectivisation des problèmes, la défense du client, ${ }^{2}$ le questionnement de l'idéologie dominante ainsi que l'augmentation (empowerment) du pouvoir du client.

\section{La matérialisation et la collectivisation des problèmes}

La matérialisation et la collectivisation des problèmes sont des pratiques indissociables qui exigent de l'intervenant une perception matérialiste, collective, historique et conflictuelle des problèmes sociaux. Opérationnellement, il s'agit de réfléchir et de se documenter sur les liens entre l'organisation sociale (politique, économique et idéologique), les rapports dominants-dominés qu'elle produit, et le développement de la personnalité (Sève, 1979 ; Leonard, 1984). Considérons par exemple 
différents aspects du vécu des femmes : double charge de travail, rejet vécu par plusieurs d'entre elles quand elles réussissent dans la vie publique, blâme qui leur revient quand surgissent des problèmes avec leurs enfants et dans leur mariage, socialisation à la coopération, à l'abnégation passive au service des autres, plus grande tendance à développer des comportements étiquetés « névrosés ", comme la dépression. De même, la "surprotection " maternelle est comprise comme un désir possible de la femme de prolonger son utilité sociale, étant donné que la maternité peut être la seule source de valeur et de reconnaissance sociale.

Une perspective matérielle et collective exige de connaître la « théorie de l'attribution " indiquant que les échecs des femmes sont plus sujets à être expliqués en termes psychologiques. Quand un homme ne réussit pas dans telle ou telle situation, la plupart des gens, indépendamment de leur classe sociale, attribuent en effet cet échec à des facteurs externes. Par contre, quand une femme ne réussit pas dans une situation semblable, son échec est d'habitude expliqué par des facteurs internes. À l'opposé,le succès des hommes est typiquement attribué à des facteurs internes (il est intelligent) alors que le succès des femmes est interprété par des facteurs externes (c'était un examen facile) (Caplan, 1987: 225). Les clientes sont donc possiblement plus portées à psychologiser leurs problèmes que les clients.

Un intervenant qui matérialise et collectivise évite de considérer a priori la soumission et la passivité comme des comportements sains chez les femmes. Il comprend que si plusieurs d'entre elles ont recours à des façons détournées et à du sabotage psychologique pour obtenir ce qu'elles veulent des hommes, ce sont souvent leurs seuls moyens d'exprimer leurs frustrations et ressentiments vis-à-vis de leur statut moins élevé.

Dans l'intervention auprès des hommes, adopter une vision matérialiste et collective des problèmes exige d'être conscient des récompenses sociales qu'on accorde à leurs comportements de domination, de contrôle et de compétition envers les autres. Il s'agit d'être sensible aux liens entre leur socialisation à la négation des sentiments et des émotions (les leurs et ceux des autres), leurs tendances à objectifier les autres et à s'exprimer par la violence, et le fait que leurs comportements sont plus susceptibles d'être étiquetés "sociopathes" ou "psychotiques".

Le travail auprès des hommes nécessite aussi que l'on soit particulièrement attentif d'une part, à leurs conditions de travail et à la signification qu'ils leur accordent, et d'autre part, aux liens possibles entre une certaine impuissance vécue dans le milieu du travail et, en guise de compensation, une tendance à abuser de leur pouvoir au foyer. II s'agit 
de comprendre qu'être commandé toute sa vie, ça " marque son homme", y compris dans les images parentales que les enfants portent dans leur inconscient (Mispelblom, 1982: 178).

Dans l'évaluation et l'intervention sociales, on veut préciser dans quelle mesure une cliente est considérée de moindre valeur et ainsi traitée, dans quelle mesure elle est l'objet de discrimination au travail sur la base de caractéristiques socio-démographiques: sexe, statut civil, orientation sexuelle, âge, religion, santé, race, ethnie, nationalité ou appartenance de classe.

L'intervenant qui veut être matérialiste et collectif doit éviter de redéfinir la situation d'une cliente dans la seule perspective des ressources offertes par l'organisme employeur. Quand les problèmes sont d'ordre financier, du domaine du logement ou sont dus à une difficulté d'accès à un travail valorisant et payant, l'intervention doit aller au-delà de l'exploration des sentiments. Par ailleurs, prenons l'exemple d'un homme qui violente sa femme. Il faut veiller, dans le travail avec ce couple, à ne pas centrer l'intervention sur des changements que la femme aurait à faire, sous prétexte qu'il est plus difficile de rejoindre un homme affectivement. Il faut donc éviter, dans ce cas, d'orienter la femme vers une modification de ses attentes, une modération de son agressivité, une plus grande sensibilité aux humeurs et désirs de son mari. Agir ainsi équivaudrait à lui suggérer de se conformer aux attentes du mari et aux comportements que celui-ci tente d'imposer par sa force physique.

L'intervenant matérialiste et collectif s'oppose à la théorie dominante qui considère inévitablement l'inceste comme un symptôme de dysfonctionnement familial au niveau des rôles. Une telle définition de l'inceste excuse l'agresseur et renforce les rôles traditionnels, au détriment des femmes. Assigner un rôle à chaque membre de la famille, dans une situation incestueuse, revient à rendre femmes et enfants coupables de ce qui leur arrive. La victime de l'inceste devient ainsi la responsable, et le phénomène incestueux lui-même est interprété comme une intercommunicatioon sophistiquée entre deux ou trois victimes dans le contexte familial - comme si victimes et bourreau jouissaient d'un pouvoir égal!

Intervenir auprès de parents par le biais de la Protection de la Jeunesse, dans une perspective matérialiste et collective, amène l'intervenant à démasquer avec eux le rôle de l'État et de ses fonctionnaires, ainsi que le rôle des employeurs dans les situations de négligence dans lesquelles se trouvent beaucoup d'enfants. Même si l'État rend les parents légalement responsables de l'éducation de leurs enfants, c'est lui et les employeurs qui contrôlent principalement les conditions matérielles d'existence de la famille, par le biais des salaires ou des prestations 
versés. Les effets de l'exploitation du milieu de travail à l'intérieur de la famille comptent aussi pour beaucoup dans la négligence des enfants.

Dans son travail auprès de clientes de la classe ouvrière (clientèle majoritaire des services sociaux), être sensible à une telle perspective amène l'intervenante à comprendre le cynisme, la méfiance et la distance que peuvent manifester plusieurs envers elle. Ces femmes, conscientes de la mentalité du "chacun pour soi", savent que dans notre monde, personne, même l'intervenante, n'agit sans intérêts personnels. Celle-ci est aussi consciente du fait que la plupart des organismes sociaux sont moins accessibles aux clientèles plus démunies économiquement : une femme peut avoir à faire de longs trajets, dépendre des autres pour ses déplacements, ne pas avoir les moyens financiers pour payer le transport et/ou le gardiennage qu'implique un rendez-vous. De son côté, un homme qui veut consulter doit obtenir la permission de son patron pour s'absenter du travail, et son salaire est réduit en conséquence. Concrètement donc, il faut être disponible aux moments qui conviennent à ces clients (soir et fin de semaine), et être assez flexible pour leur permettre de se présenter, à l'occasion, sans rendez-vous.

Enfin, l'intervenante matérialiste et collective demeure critique visà-vis toute approche ou modèle d'intervention incapable de considérer les causes sociales des problèmes individuels. Elle s'oppose donc aux approches qui supposent que ce sont uniquement et avant tout des changements au niveau personnel ou familial qui, en dernier lieu, transforment les conditions matérielles objectives des clients. Pour adopter une telle vision, il faut s'habituer à définir la situation d'un client en termes collectifs plutôt qu'uniquement en termes individuels (Walker et Beaumont, 1981). Quand le client parle, il faut avoir automatiquement en tête qui il est socialement, c'est-à-dire politiquement, économiquement et idéologiquement, et se demander s'il est socialement infériorisé à cause de l'une ou l'autre de ces caractéristiques socio-démographiques: race, ethnie, nationalité, sexe, classe sociale, religion, santé, âge, statut civil et orientation sexuelle. Il faut explorer les similitudes entre le vécu de tel client et le vécu des personnes qui occupent des statuts identiques. En entrevue, au lieu de simplement refléter les idées et sentiments d'un client, l'intervenant structurel veillera à ajouter une dimension sociale à son expression d'empathie (Keefe, 1980 ; Hepworth et Larsen, 1982; Fook, 1986). Utilisant la technique d'universalisation (Carey-Bélanger, 1974), il reflétera les liens entre les sentiments, idées ou comportements adoptés par le client et les statuts sociaux qu'il occupe. Fook (1986: 58) illustre bien la différence entre faire preuve d'empathie sociale et démontrer une empathie personnelle envers une cliente. À une ménagère ennuyée et déprimée, s'exclamant que "les hommes finissent toujours 
par avoir ce qu'ils veulent ", au lieu de limiter le reflet à : " vous sentez que vous n'arrivez jamais à obtenir ce que vous désirez ", l'intervenante ajouta : " et vous croyez que les hommes finissent toujours par s'en sortir mieux que les femmes ", soulignant ainsi les perceptions de la cliente de sa condition sociale.

Tant à l'intérieur qu'à l'extérieur de l'organisme employeur, il s'agit aussi pour l'intervenant de favoriser des contacts entre des personnes qui vivent des situations similaires. On tente de promouvoir une collaboration et un échange de services entre clients et si possible, de favoriser une remise en question collective des structures qui les oppriment. On est critique face à toute intervention individuelle ou de groupe qui ne jumelle pas à la provision de services une certaine forme de mobilisation en vue de revendiquer plus de ressources (Leonard, 1975). Par exemple, un intervenant regroupa, pour des fins d'entraide et de support, des clients sérieusement handicapés d'un institut de réadaptation. II les amena à s'interroger sur le modèle médical et individuel dominant de l'institut même. Il fit connaître et propagea une approche d'intervention plus structurelle et axée sur une philosophie d'existence prônant l'indépendance. II recadra ainsi les problèmes de ses clients d'une perspective individuelle et strictement personnelle à une perspective collective, plus matérielle et sociale. Depuis, le groupe s'est détaché de l'institut. Avec le support "silencieux" de l'intervenant, l'institut est devenu, pour le groupe, une cible d'intervention privilégiée: manque d'orientation communautaire, pénurie de ressources matérielles pour les clients, manque de personnel, etc.

Les structures de service qui permettent et encouragent le travail de groupe, plutôt qu'uniquement l'intervention individuelle, sont également favorisées. Concrètement, on tente d'abord d'intervenir en groupe avec les clients. Bien sûr, il ne s'agit pas d'éliminer l'approche individuelle, mais plutôt de remettre en question son exclusivité. Dans cette optique, l'intervention individuelle auprès d'un client vise sa participation éventuelle à un groupe. Pour ce faire, on favorise l'assignation des dossiers par zone géographique ou écologique (un "cas" devient ainsi une rue, un étage dans un centre hospitalier, un pâté de maisons, etc.). Les visites à domicile et dans le milieu sont aussi encouragées, afin de maximiser la compréhension de l'intervenante des conditions objectives dans lesquelles vit le client.

Quand c'est possible, on tient compte, dans l'assignation des dossiers, des similitudes entre la cliente et l'intervenante. Quand celle-ci et la cliente appartiennent à la même collectivité ou ont vécu des expériences semblables, l'intervenante partage son vécu personnel, dans la mesure où cette attitude peut aider à redéfinir, en termes matériels et/ou 
collectifs, la situation-problème de la cliente. Une vue matérialiste et collective implique, pour l'intervenante, une prise de conscience de ses propres collectivités d'appartenance, institutionnelle et de classe. Cela permet de préciser dans quelle mesure et sur quelles bases concrètes on peut établir une alliance consensuelle ou conflictuelle avec la cliente. Par exemple, dans son contrat avec un couple, un intervenant indiqua qu'étant homme, il risquait peut-être de s'identifier plus facilement et plus spontanément à l'époux. Par ailleurs, il ajouta qu'il aurait peut-être plus à défendre l'épouse, compte tenu du statut inférieur des femmes à l'intérieur et à l'extérieur de la famille.

Il est nécessaire de bien connaître le rôle social de l'organisme employeur vis-à-vis les collectivités avec lesquelles on travaille, de se familiariser avec les contradictions possibles entre le rôle officiel de l'organisme et son rôle réel, de connaître les limites, les choix et les possibilités d'intervention imposés par l'encadrement organisationnel (Forbes, 1986). Pratiquement, il s'agit de clarifier dans le contrat les enjeux conflictuels possibles entre les intérêts du client, ceux de l'intervenant et ceux de l'organisme (Cingolani, 1984; Bourgon, 1988). Par exemple, dans son travail auprès d'une cliente qui, à l'insu du Service de l'aide sociale, vivait avec son ami et recevait des prestations tout en occupant un emploi à temps partiel afin d'arrondir ses fins de mois, une intervenante de la Protection de la jeunesse promit qu'elle "ferait l'aveugle» devant ces infractions, mais qu'elle ne pouvait passer sous silence la négligence de la cliente qui laissait constamment son jeune enfant, seul, le soir.

L'intervenant soucieux d'adopter une perspective de pratique matérialiste pourrait être appelé à exposer le rôle et le fonctionnement de l'organisme relativement au problème du client. Il pourrait même avoir à exprimer son désaccord avec la façon privilégiée par l'organisme pour définir et résoudre la difficulté de la cliente. Une travailleuse sociale en milieu scolaire fit remarquer à sa cliente, mère d'un garçon renvoyé de l'école pour s'être battu avec un autre enfant, que l'école elle-même encourageait les enfants à voir la coercition physique comme nécessaire et désirable dans la résolution des différences entre les personnes, puisque légalement, on y punissait encore les enfants avec le martinet (courroie de cuir).

La pratique matérialiste et collective exige parfois de mettre en relation des faits qui, à prime abord, pourraient ne pas paraître reliés. II s'agit d'explorer avec la cliente les liens entre sa situation actuelle et différents autres facteurs : son identité personnelle et collective, ses conditions de travail et de vie, les valeurs et idéologies sociales de son milieu, la façon dont l'entourage perçoit son problème. Nous voulons 
ainsi démarquer la responsabilité individuelle et la responsabilité sociale dans la situation d'une personne, c'est-à-dire évaluer objectivement dans quelle mesure une cliente est socialement placée dans telle situation, et dans quelle mesure elle y contribue par sa propre soumission ou son évasion plutôt que par l'affirmation et la confrontation.

Quand on sait comment les structures sociales ont tendance à disqualifier le point de vue et l'expérience de ceux qui les questionnent ou s'y opposent, on n'est pas surpris de la tendance de plusieurs clients à considérer leur réalité de façon fataliste, naturelle et donc inchangeable. Il faut aussi travailler à modifier cette attitude.

On explore donc les liens entre la socialisation, les choix réels de la cliente compte tenu des biais sociaux à son égard, et ses façons de penser, de sentir et d'agir. On explore le rôle des croyances, attitudes et comportements d'autrui dans le renforcement négatif de l'image de soi et la tendance à personnaliser ses échecs. Par exemple, avec une femme qui assume seule la responsabilité de ses enfants, on fouille les liens entre cette situation sociale et ses sentiments d'appréhension, ou sa tendance à les surprotéger. Avec une femme battue, on établit les liens entre sa peur et son insécurité de se retrouver seule, sans ressources et peut-être stigmatisée socialement, et son désir de retourner vivre avec son agresseur (René, 1987). Il s'agit de cerner l'interprétation que fait la cliente de sa propre situation, et de compléter cette analyse avec elle, en y ajoutant les éléments qui permettent de faire ressortir le rôle déterminant des conditions objectives dans la situation. On veut ainsi éviter d'interpréter les problèmes uniquement par des facteurs individuels - conscientiser afin de déculpabiliser, normaliser et déresponsabiliser le client qui, injustement, se définit comme le seul responsable d'une situation qui ne relève pas exclusivement de lui.

Il est important de souligner l'attention particulière accordée à ce processus dans l'approche structurelle. Il s'agit de saisir la signification totale, personnelle et subjective que le client accorde à ses expériences individuelles et sociales. Concrètement, c'est en complétant avec la cliente sa socio-biographie historique (Mispelblom, 1982) que l'intervenante et la cliente établissent les liens possibles entre, d'un côté, les exigences, restrictions et choix sociaux vécus objectivement et historiquement par la cliente dans sa famille et dans d'autres institutions, et, d'un autre côté, sa santé, ses idées, comportements et sentiments envers elle-même et les autres. Le génogramme (Guerin et Pendagrast, 1976) et la carte de réseau (Speck et Rueveni, 1969) sont des outils d'intervention indispensables pour amorcer ce processus.

Une attention particulière est portée aux expériences passées ou présentes de conflits sociaux vécus par la cliente. On vérifie dans quelle 
mesure elle a pu être sujette à des expériences d'exploitation, d'impuissance, $d$ 'isolement ou de dépendance dans sa vie familiale et sociale. On tente de comprendre les liens entre ses expériences de vie personnelle et sociale, son estime de soi, ses désirs, ses douleurs non exprimées, ses peurs non résolues, ses espoirs personnels, son contact affectif avec ellemême, sa capacité d'identification sociale et la possibilité qu'elle soit identifiée à ses oppresseurs.

On est à l'écoute afin d'identifier des scénarios de vie ou "scripts" (Steiner, 1974) et contrats conscients ou inconscients qu'a pu adopter la cliente envers elle-même et/ou les autres, compte tenu de ses expériences personnelles et sociales. On est attentif au rôle de l'imaginaire dans le maintien de son problème, aux liens possibles entre ses pensées autoprotectrices et son incapacité ou refus d'agir constructivement.

La technique d'individualisation contextuelle (Caplan, 1987) peut servir à attirer l'attention de la cliente sur la spécificité de sa situation quand elle est portée à se comparer injustement à d'autres qui vivent dans un contexte différent. En d'autres mots, il ne suffit pas de collectiviser une situation avec un client et de comprendre comment ses expériences ressemblent à celles des autres, mais il faut aussi saisir ce qui lui est spécifique et qui relève de sa famille ou de son tempérament, et qui explique sa manière d'intérioriser les messages sociaux. Ce genre d'exploration peut amener l'intervenante à remettre en question avec la cliente sa perception d'elle-même et de sa situation. Pour ce faire, on peut utiliser la technique du questionnement critique (Moreau, 1979; Fook, 1986), qui consiste à réfléchir avec la cliente sur le pourquoi social, c'est-à-dire le pourquoi politique, économique et idéologique d'une situation. Utilisant une variété de moyens tels l'humour dialectique (Frayne, 1987), les métaphores (Gordon, 1978), les histoires, l'utilisation de la dissonance cognitive (Festinger, 1957), la vérification des inférences (Middleman et Goldberg, 1974), I'imagerie mentale (Erickson, 1982), la persuasion (Simons, 1982) et même le silence (Bourgon, 1988), l'intervenante aidera le client à recadrer la signification qu'il accorde à sa situation. Par exemple, l'agressivité et la rage des personnes socialement dévalorisées pourront être reconnues comme valables pour leur autoprotection immédiate et ainsi réétiquetées positivement. On pourra, par exemple, exprimer à une femme étiquetée négativement comme dépendante à cause de son besoin d'affection et de son intérêt aux sentiments des autres, que ce comportement peut être interprété comme une capacité d'entrer en relation avec les autres et d'investir auprès d'eux.

L'intervenante se demandera avec la cliente pourquoi les idées, comportements ou sentiments de cette dernière sont jugés socialement indésirables, qui les juge ainsi, au profit de qui, quels seraient les 
avantages et désavantages de questionner et de contrer cette situation, quels pouvoirs et protection seraient nécessaires pour le faire, et si, en fin de compte, elle désire le faire. Par exemple, un travailleur social intervenant auprès d'un client paraplégique, déprimé du renversement de rôles et de sa perte de capacité sexuelle qu'avait entraînés son handicap, l'amena à remettre en question sa définition des rôles sexuels traditionnels. Il lui fit voir qu'il pourrait profiter de son temps à la maison pour se rapprocher de ses deux enfants, distance affective dont il se plaignait par ailleurs, et il le référa à un sexologue pour obtenir de l'information sur des façons alternatives de performer sexuellement.

Décortiquer les liens entre le vécu personnel familial, l'idéologie dominante et les conditions objectives de la famille permet de démêler les problèmes qui proviennent de l'extérieur de la famille, de ceux qui sont liés à des tentatives présentes de dénouer des expériences passées et non résolues. II s'agit d'empêcher que la famille soit injustement tenue l'unique responsable de situations qui dépendent aussi de facteurs qui lui sont externes. Ce processus peut amener la cliente à vivre ou à revivre des sentiments pénibles, parfois longuement refoulés, dans la mesure où son vécu familial fut une source immédiate d'oppression pour elle. La famille étant dans notre société l'institution où souvent se jouent beaucoup de restrictions sociales au niveau du sexe, de l'âge et de la classe sociale, il n'est pas surprenant qu'elle soit perçue et vécue par plusieurs comme une prison plutôt qu'un refuge (Lasch, 1979).

Ce n'est souvent qu'après avoir pu suffisamment ventiler des sentiments refoulés de peur, d'agressivité ou de douleur, associés possiblement à son vécu familial, qu'une personne pourra en arriver à saisir autrement son vécu, par une compréhension différente de ses parents et de leur situation, qu'elle pourra peut-être aller au-delà d'une définition familialiste des problèmes et, quand cette attitude est appropriée, parvenir au pardon (Moreau, 1978 ; Wyckoff, 1977 ; Lerner, 1977). Il est important de souligner que la pratique de matérialiser et de collectiviser une situation avec un client peut être un processus pénible et de longue haleine. C'est pourquoi ce travail est plus facilement entrepris en intervention de groupe qu'en intervention individuelle.

Enfin, la pratique matérialiste et collective exige d'accorder une priorité à l'aide matérielle, de maximiser l'accès concret aux ressources, incluant les ressources de l'organisme employeur. Par exemple, dans la Protection de la jeunesse, on veille d'abord à ce qu'une cliente ait suffisamment d'argent et ait accès à une garderie ou à de l'aide à la maison, avant d'entreprendre avec elle des changements aux niveaux de son état émotif, de ses idées ou de ses comportements. 


\section{La défense du client}

L'intervenante structurelle pourrait être appelée à défendre les intérêts de sa cliente à l'intérieur et à l'extérieur de l'organisme qui l'emploie, dans la mesure où l'insuffisance des ressources des établissements visés contribue à la création ou au maintien d'une situation problème. Utilisant les techniques de courtage, de médiation et d'avocat (Middleman et Goldberg, 1974), un des soucis premiers sera de favoriser l'utilisation maximale des ressources par la cliente, tout en minimisant l'impact négatif que peut représenter une telle utilisation. Cette pratique s'oppose carrément à celle exigée officiellement de la plupart des intervenantes sociales, à savoir contribuer à rationaliser et à réduire l'utilisation des ressources sociales existantes. Privilégiant l'universalité des services, l'approche structurelle incite le plus grand nombre de clientes éligibles à utiliser les services, tout en veillant à ce que leur situation n'empire pas. Cette vision suppose une compréhension conflictuelle des enjeux impliqués dans les services sociaux, considérés comme des lieux de débats auxquels tant le client et l'intervenant peuvent contribuer.

L'utilisation maximale des ressources (incluant celles de son organisme employeur) exige de l'intervenante une connaissance des codes de lois civiles et municipales et des principaux organismes sociaux : aide sociale, C.L.S.C., D.P.J., écoles, hôpitaux, centres d'accueil et même, services d'utilité publique tels Bell Téléphone, Hydro-Québec et Gaz Métropolitain. Elle doit surtout être familière avec leurs rôles parfois contradictoires, leurs exigences, procédures, pratiques, idéologies et conditions de service. Quand pour une cliente, l'utilisation d'une ressource risque de causer ou d'aggraver un problème, l'intervenante fera tous les efforts pour contrer cette situation. Concrètement, elle ne référera jamais à la légère une cliente à un autre intervenant ou à une autre ressource. Elle en profitera pour examiner et discuter avec elle les limites autant que les mérites de la ressource, et pour exposer la relation qui sera exigée de la cliente en l'utilisant. Par exemple, sachant que ses comportements risquent d'être étiquetés masochistes par telle ressource, on la mettra en garde et on la préparera à questionner cette définition de sa situation. L'intervenante personnalise les références. Elle ne réfère personne à un organisme tout court mais plutôt, si possible, à une personne précise à l'intérieur de cet organisme. Elle s'assure ensuite d'un "feedback" de la cliente sur la pertinence et la qualité de la ressource à laquelle on l'a référée. Une attention particulière est portée aux transferts de dossiers entre organismes ou intervenants. Idéalement, elle verra à que ce transfert se fasse en présence des personnes concernées : cliente, intervenante qui réfère et intervenante qui accueille. Si 
cette pratique est impossible, l'intervenante veille minimalement à ce que le dossier documente le rôle de l'insuffisance des ressources dans une situation problème.

Dans le cas où l'on découvre qu'une cliente a été envoyée au mauvais endroit, ou que l'utilisation de l'organisme employeur par la cliente lui cause une difficulté, on ne néglige pas de communiquer avec la personne ou l'organisme référant, d'abord pour les informer à ce sujet, puis pour éviter que d'autres personnes soient exposées à une telle situation. Par exemple, une jeune mère célibataire et son bébé furent référés par un médecin à l'Urgence d'un centre hospitalier plutôt qu'à la Protection de la jeunesse. Le problème de la mère en était essentiellement un d'habitation (son logement ne comprenait qu'une chambre à coucher et son co-locataire ne pouvait plus supporter les pleurs de l'enfant.) Le résultat fut que la situation de cette cliente fut pathologisée à outrance par les médecins. On la référa en psychiatrie et on lui prescrivit des tranquillisants malgré la protestation de la travailleuse sociale. Ce n'est qu'en recontactant le médecin référant, pour l'informer des services de la Protection de la jeunesse, tels le placement, qu'avec l'appui de ce dernier l'intervenante a pu réacheminer la cliente vers l'organisme approprié plutôt que vers la psychiatrie.

Comme le suggèrent Friedberg (1977) et d'autres (Brager, 1972; Deehy, 1977), les contradictions dans les organismes sociaux et la marge d'autonomie dont jouissent les intervenants, permettent à ces derniers d'entreprendre plus de changements intra-organisationnels qu'ils ne le pensent. Toutefois, il se peut qu'ils aient à recourir à des pratiques silencieuses (Mispelblom, 1982). Des recherches (Pearson, 1975 ; Moreau, 1987) démontrent en effet qu'il arrive fréquemment à des praticiens sociaux d'avoir recours à de telles pratiques, dont certaines pourraient même être qualifiées de "sabotage industriel ". Par exemple, des intervenantes rapportent avoir été aveugles devant des infractions légales de leurs clientes ou devant des politiques ou pratiques sociales non respectées par elles, surtout quand elles-mêmes y voyaient de l'injustice. D'autres refusent d'appliquer certaines lois en informant leurs clientes des façons et moyens de les contourner.

L'intervenante peut aussi être amenée à détourner une cliente d'une ressource qui lui serait problématique. Par exemple, sachant qu'une référence à tel médecin risque d'empirer l'image qu'a la cliente d'elle-même, comme malade, ou risque de psychologiser et de personnaliser davantage sa situation sociale, elle pourra la réorienter vers une alternative, par exemple un centre de santé pour femmes qui favorise une approche plus douce, personnalisante et moins pathologique. L'accueil occupe un poste de choix pour ce genre d'intervention. Là où 
des ressources alternatives moins problématiques n'existent pas, l'intervenante travaillera à long terme à les mettre sur pied, soit en démontrant le besoin de telles ressources, soit en encourageant leur développement.

La défense du client exige d'être attentif à l'état des informations déjà recueillies. Tantôt, défendre consistera à augmenter la visibilité d'une situation, à faire ressortir publiquement les liens entre telle situation sociale et les problèmes personnels conséquents. Tantôt, défendre consistera plutôt à diminuer la visibilité d'un fait, soit en omettant d'écrire ou de souligner certains événements au dossier, soit en y réétiquetant une situation. Par exemple, ayant remarqué en lisant le dossier d'une de ses clientes à la Protection de la jeunesse, qu'au cours des années, elle avait subi de nombreux problèmes matériels et institutionnels définis et étiquetés comme étant d'ordre personnel, une intervenante profita de l'occasion de la fermeture du dossier pour noter dans son résumé les véritables causes de la situation de cette cliente. Ce geste lui évitera possiblement d'être stigmatisée si jamais son dossier est réouvert.

La défense exige aussi d'intervenir plus souvent de façon directe et active. Par exemple, on sera moins hésitant à agir comme intermédiaire ou accompagnateur d'un client, si cela peut l'aider. Bien sûr, on favorise d'abord le développement des capacités d'auto-défense du client face aux institutions. À cet égard, on travaille à améliorer les capacités d'écoute, d'affirmation, de négociation et de persuasion du client. L'intervenant qui défend ses clients est aussi celui qui soigne son langage (Edelman, 1974) à leur égard, adapte son langage au leur et évite de les dépersonnaliser par l'appellation qu'il leur donne.

Recadrer la signification et recadrer contextuellement (Bandler, 1982) sont deux techniques utiles. Par exemple, pour défendre une cliente devant un organisme ou un individu qui lui cause des problèmes, l'intervenante peut devoir recadrer positivement avec elle ou d'autres personnes, la signification de ses sentiments, comportements ou idées jugés problématiques, de façon à identifier et à souligner leurs aspects positifs. Quant au recadrage contextuel, il consiste à identifier et faciliter l'accès de la cliente aux lieux, circonstances, structures, où ses idées, sentiments ou comportements seraient considérés acceptables sinon souhaitables. L'intervenante cherchera enfin à utiliser toutes occasions pour démontrer son respect envers la cliente et favoriser ainsi une meilleure estime d'elle-même.

Il ne faut pas sous-estimer le pouvoir de récupération et de coercition des services sociaux organisés (Patti et Resnick, 1972). Il faut bien choisir les luttes intra-organisationnelles dans lesquelles on s'implique, parce 
que toutes ne valent pas qu'on y consacre des énergies. II faut surtout bien mesurer et bâtir le pouvoir nécessaire pour mener une lutte institutionnelle (le pouvoir légitime, de nombre, de référence, d'information et de coercition) (French et Raven, 1965). Généralement, on n'entreprend jamais seul une telle lutte, d'où l'importance de cultiver ses alliances, de se syndiquer si on ne l'est pas et, au besoin, d'utiliser son syndicat comme appui collectif. Dans la mesure du possible, c'est au client que reviendra toujours d'accepter ou non qu'une intervenante le défende.

\section{Le questionnement de l'idéologie dominante}

Défendre la cliente implique, pour la praticienne, d'être attentive à ses propres comportements, sentiments et idées qui pourraient potentiellement contribuer à l'oppression de cette cliente. (Walker et Beaumont, 1981). C'est veiller à ne pas renforcer ou reproduire l'idéologie et les rapports sociaux dominants à l'intérieur ou à l'extérieur de ses interventions. Concrètement, on se garde d'être sexiste et familialiste. En "thérapie " familiale par exemple, on évite particulièrement de surresponsabiliser les femmes ; l'absence paternelle est considérée tout aussi significative que l'omniprésence maternelle dans le problème d'un enfant (Caplan, 1985); on appuie les femmes qui désirent travailler à l'extérieur du foyer, tout en reconnaissant et en remettant en question avec elles leur double charge de travail ; les soins maternels à l'intérieur de la famille ne sont pas automatiquement perçus comme les seuls valables et les meilleurs pour les enfants et les personnes âgées; la valeur des personnes n'est pas associée à leur capacité ou à leur désir d'avoir des enfants (Rich, 1981). Concrètement, cela exige de se garder de renforcer la maternité dans son travail auprès de femmes pour qui elle fut un choix imposé (Levine et Estable, 1982). Cela peut impliquer le support au droit des femmes du contrôle sur leur corps, au droit à l'avortement ou à des méthodes de contraception saines; pour celles devenues mères malgré leur volonté, il s'agit de les aider à envisager librement et sans culpabilité la possibilité de l'adoption.

Toutes les formes de la famille, et non seulement la famille biparentale, hétérosexuelle, isolée, nucléaire, représentée par l'homme unique ou principal pourvoyeur, et la femme à la maison ou gagne-pain secondaire, sont encouragées, tant concrètement qu'au niveau des politiques sociales. Cela implique l'appui à l'universalisation de l'accès aux garderies et la création de plus de refuges pour femmes et enfants victimisés. 
Quant aux personnes âgées, aux jeunes et aux personnes handicapées, on questionne avec elles toutes formes d'exclusion et de traitement différent à leur égard. On évite, en particulier avec les enfants, de leur adresser la parole comme s'ils étaient sourds ou imbéciles et de leur imposer un contact physique, comme si leur intimité ne comptait pour rien. On se garde d'interpréter la tendance qu'ont certaines personnes âgées à revenir sur leur passé, comme un signe évident de confusion. Comme le souligne Pincus (1970), si une personne âgée interviewée par une personne plus jeune perçoit leur différence d'âge comme une menace à son statut, elle peut utiliser ce mécanisme de recul dans le temps pour tenter d'effacer cette différence.

Ne pas reproduire l'idéologie dominante, c'est aussi dissocier la valeur et la santé des personnes de leur capacité et volonté de travailler. Il s'agit de ne pas renforcer l'éthique du travail surtout dans un contexte de chômage (Walker et Beaumont, 1981). Il n'y a pas une relation automatique entre la position occupée par une personne dans la division sociale du travail et ses capacités innées. On se garde enfin d'être raciste ou ethnocentrique (Turner, 1972) dans ses interventions. Par exemple, de nombreuses recherches démontrent que l'importance accordée à la famille, à la religion, à la verbalisation, au silence, au contact par les yeux, et même aux distances physiques considérées confortables entre les personnes, varie considérablement selon les cultures (Sue et Sue, 1977). On doit donc se garder d'imposer le modèle culturel dominant au sein de ses interventions.

\section{L'augmentation du pouvoir du client}

Augmenter le pouvoir de la cliente consiste à lui fournir les occasions d'un contrôle maximal de l'interaction cliente-organismeintervenante. Pratiquement, on veille d'abord à l'aider à agir elle-même avant d'agir avec ou pour elle (Hashimi, 1981). On insiste sur son droit à assister aux discussions de cas qui la concernent. On tente de rendre ses interventions aussi transparentes que possible. Par exemple, le contenu du dossier d'une cliente est partagé et même rédigé avec elle. On évite des stratégies cachées, dans ses entrevues. On expose le rationnel derrière ses questions. L'utilisation d'une technique ou d'une stratégie d'intervention sert d'occasion de démystification. On discute, par exemple, les origines et fondements d'une technique d'intervention, on identifie d'autres situations où celle-ci pourrait servir et on encourage la cliente à partager ces connaissances avec d'autres (Bilodeau, 1980). En animation de groupe, on tente d'intervenir de façon à augmenter la 
fréquence des interactions entre les membres, plutôt qu'entre les membres et l'animateur.

L'augmentation du pouvoir de la cliente favorise l'établissement d'une relation dialogique (Freire, 1973) avec elle - c'est-à-dire caractérisée par un rapport horizontal plutôt que vertical. Le rapport aidant-aidée est compris comme un échange de significations entre personnes (Stephen et Black, 1985) - d'où l'importance pour l'intervenante de se garder d'imposer son propre point de vue à partir de son expérience de vie personnelle, et dans la mesure du possible, de valider le point de vue de la cliente (Caplan, 1987).

Au lieu de s'afficher comme expert, l'intervenant se met en position d'apprentissage mutuel avec le client. Il tente d'aider à définir, à poser et à contextualiser les problèmes, tout en favorisant le questionnement et l'expression d'un désaccord; il expose ses propres incertitudes à l'égard de ses interventions (Stephen et Black, 1985 ; Schon, 1983, 1987). Concrètement, il essaie de réduire, sans la masquer, la distance sociale entre eux, que ce soit au niveau du langage, de l'habillement, ou par d'autres moyens.

Enfin, l'augmentation du pouvoir vise le développement des capacités du client d'agir sur sa situation. On identifie et on travaille à modifier les idées, sentiments et comportements du client qui l'empêchent de changer sa situation ou qui contribuent au maintien d'une situation problématique pour lui ou pour les autres (Adam, 1978; Nelson-Jones, 1984). On s'assure que le client est réaliste dans son attribution de la responsabilité, dans son anticipation des risques et des gains impliqués, dans les règles et standards personnels qu'il adopte et dans ses attentes face aux autres. On améliore ses capacités de développer des explications alternatives dans une situation. On l'aide à distinguer entre la réalité et ses fantasmes ou interprétations parfois erronées de cette réalité. On l'aide à identifier et, au besoin, à contrer les idéologies qui contribuent à son inertie. On travaille avec lui les distortions, rationalisations, négations, identifications ou projections auxquelles il a peut-être recours et qui servent, soit à le garder dans l'inaction, soit à opprimer les autres. On remet en question avec lui ses tendances à s'auto-étiqueter négativement. Quand cela est approprié, on identifie avec le client les gains secondaires qu'il retire peut-être de sa contribution personnelle à son oppression. On examine ces avantages implicites et on tente d'affaiblir leur force d'action. On améliore ainsi ses capacités d'analyse, de prise de décision, de planification, d'action et d'évaluation.

Au niveau affectif, on travaille à augmenter la sensibilité du client à ses propres émotions, on l'aide à identifier avec justesse ses émotions, à se les approprier, à contrôler leur expression et à les mobiliser afin 
d'agir constructivement. L'intervention auprès des femmes, par exemple, souligne leur droit à la séparation d'avec le conjoint. On y travaille également le droit de ressentir et d'exprimer un sentiment légitime d'agressivité, au lieu de le refouler et de vivre dans la culpabilité (Mueller et Leidig, 1976). Toujours on cultive l'estime, l'acceptation et l'amour de soi.

Au niveau des comportements, on travaille à améliorer les habiletés d'affirmation, de négociation et de gestion de conflit. On aide le client à améliorer ses habiletés de communication verbale et non-verbale, d'écoute, de réceptivité aux réactions des autres, de même qu'à améliorer ses capacités de partager son vécu. Dans les cas où un individu se révèle incapable d'empathie sociale ou incapable de s'identifier aux autres en tant que personne humaine, et que cette inaptitude contribue à faire vivre de l'oppression à d'autres, des efforts sont alors faits pour réduire la distance sociale entre eux. Quand le client traîne des ressentiments qui l'empêchent d'agir constructivement pour lui-même, ou qui contribuent à détruire les autres, on l'aide à identifier et à analyser les origines sociales de ces sentiments. L'imagerie mentale (Erickson, 1982), le psychodrame (Maier et al., 1975) et la discussion logique sont des techniques qui peuvent favoriser ce travail. Enfin, on l'aide à reconnaître et à éliminer les comportements qui provoquent le rejet et contribuent ultimement à une baisse d'estime de soi.

\section{Conclusion}

L'application de l'approche structurelle n'est pas sans soulever des contradictions et dilemmes importants dans la pratique (Lamont, 1987). Les problèmes d'éthique liés à la défense du client sont complexes. Qui défend-on quand on intervient dans un groupe comme la famille et que, dépendant du point de vue, tous les membres y sont opprimés ? Sur quoi se base-t-on pour prendre une telle décision? Quand la défense d'un client devient-elle une forme de contrôle plus subtile, une sorte de paternalisme ou de maternage à son égard? Comment défendre le client sans affaiblir son pouvoir ? Comment discuter avec le client du rapport qu'il croit avoir avec sa situation, et de la définition qu'il en donne, sans lui imposer sa réalité ? Que faire devant une cliente qui refuse de remettre en question son pouvoir et qui insiste pour que l'intervenante maintienne le sien? Comment conserver une crédibilité et ne pas provoquer la désorganisation du client, sil'on met ouvertement en doute sa compétence devant lui ? Autant de questions épineuses et difficiles. 
Toutes les pratiques de l'approche structurelle sont intimement liées. De toute évidence, cette approche requiert des habiletés tant dans l'intervention individuelle que familiale et communautaire. Elle n'est pas un dogme, mais une hypothèse de travail qui, pour demeurer pertinente et valable dans l'intervention sociale, doit être continuellement confrontée aux tests de la pratique. Elle requiert une certaine énergie et comporte un certain risque. Par exemple, l'utilisation de certaines techniques de l'approche féministe, comme le partage du vécu personnel ou la divulgation du contenu de son dossier à une cliente, ne veut pas nécessairement dire que l'intervenante travaille dans la perspective de l'approche structurelle. Cette pratique est beaucoup plus large et exigeante : c'est une façon quotidienne de penser et d'agir à tous les niveaux (René, 1987). Face au vent de conservatisme qui a commencé à souffler récemment sur les pays occidentaux industrialisés, l'approche structurelle requiert peut-être de l'intervenant des attitudes plus douces et moins agressives.

Pour emprunter les mots de Jean Panet-Raymond (1987), peut-être faut-il, par les temps qui courent, développer une stratégie adaptée et plus souple qui sait compter sur des alliances nouvelles dans un contexte conflictuel toujours changeant.

\section{Notes}

1 Le développement de l'approche structurelle a impliqué de nombreuses personnes au cours des années. Ses bases furent établies en 1973 à l'Université Columbia de New York avec le professeur Gisèle Legault de I'Université de Montréal et le professeur Pierre Racine de l'Université du Québec à Montréal. Les professeurs Mike Brake, Peter Findlay, Roland Lecompte, Helen Levine et Allkan Moscovitch de l'Université Carleton, à Ottawa, furent également intensément impliqués. Les travaux du professeur Peter Leonard de l'Université McGill ont aussi contribué de façon importante au développement des fondements théoriques qui sous-tendent l'approche. Enfin, de nombreuses heures de discussion avec les professeurs Justin Lévesque et Jean Panet-Raymond de l'Université de Montréal, Michèle Bourgon de l'Université du Québec à Montréal, Linda Davies, Shirley Steele, Liesel Urtnowski de l'Université McGill, Gwynn Frayne, Sandra Frosst, Mary Hlywa, Marilyn Rowell du Collège Dawson à Montréal et avec Lynne Leonard, chercheure, ont servi à clarifier et à préciser ses implications pratiques. Nous leur sommes très reconnaissant.

2 Pour ne pas alourdir le texte, le masculin et le féminin sont tous deux utilisés de façon interchangeable tout au long de l'article. 


\section{Références bibliographiques}

ADAM, B., The Survival of Domination: Inferiorization and Everyday Life, New York, Elsevier, 1978.

AlthUSSER, L., "Idéologie et appareils idéologiques d'État ", La pensée, no 151, $1970: 3-38$.

Bailey, R. et M. Brake (éds.), Radical Social Work, London, Edward Arnold, 1975.

BANDler, R. et J. Grinder, Reframing, Moab (Utah), Real People Press, 1982.

BANDURA, A., Principles of Behavior Modification, New York, Holt, Rinehart and Winston, 1969.

BilodeAu, G., "Pour une réelle relation d'alliance entre travailleur social et clients ", Service social, vol. 29, no 3, 1980:438-458.

Bolger, S., P. Corrigan, J. Docking et N. Frost, Towards Socialist Welfare Work, London, MacMillan, 1981.

Bourgon, M. et N. Guberman, "How Feminism Can Take the Crazy out of your Head and Put it Back into Society", à paraître dans Women's Studies: A Canadian Perspective, édité par Géraldine Finn, Vancouver, Bergammon Press, 1988.

BRAGER, G., "Aider vs influencer : Quelques éléments politiques du changement organisationnel ", traduction de l'anglais par Justin Lévesque dans Recueil de Textes - Méthodologie de I'Intervention, École de service social, Université de Montréal, 1982 : 242-263.

BUBER, M., I and Thou, New York, Scribner's, 1958.

CaPlan, P., The Myth of Women's Masochism, New York, Signet, 1987.

CAPLAN, P., "Mother-Blaming in Major Clinical Journals", American Journal of Orthopsychiatry, vol. 55, no 3, 1985 : 345-353.

CAREY-BÉlANGER, H., "Le traitement à court terme, ses techniques et stratégies", Service social, vol. 23, nos 2-3, $1974: 57-68$.

CingolanI, J., "Social Conflict Perspective on Work with Involuntary Clients", Social Work, vol. 29, no 5, 1984 : 442-446.

DEEHY, P.T., "Les étudiants en service social et les nouvelles bureaucraties de services sociaux : l'encadrement des stratégies de survie", texte traduit de l'anglais par Maurice Moreau, présenté à la réunion annuelle de l'Association canadienne des Écoles de service social à Frédericton, Nouveau-Brunswick, 1977, $18 \mathrm{p}$.

Edelman, M., "The Political Language of the Helping Professions", Politics and Society, vol. 4, no 3, $1974:$ 285-310.

ERICKson, M.H., My Voice Will Go with You: The Teaching Tales of Milton H. Erickson, M.D., S. Rosen ed., New York, Norton, 1982.

FEStINGER, L., A Theory of Cognitive Dissonance, Stanford (Cal.) Stanford University Press, 1957.

FindlaY, P., "Critical theory and social work practice", Catalyst: A Socialist Journal of the Social Services, vol. 1, no 3, 1978: 53-68.

Fook, J., "Contributions féministes à la pratique du casework ", traduction libre par Lévesque J., Moreau M. et Panet-Raymond J. d'un texte dans Gender 
Reclaimed, Marchant, H. et B. Wearing, (éds), Sydney, Hale et Iremonger, 1986 : 54-63.

FORBES, D., "Counseling in Crisis", Catalyst: $A$ Socialist Journal of the Social Services, vol. 8, no 2, $1986: 53-84$.

Frayne, G., "Tape-Analysis Grid for the Action Research on the Structural Approach to Social Work", matériel non publié, Montréal, Université de Montréal, École de service social, 1987.

Freire, P., Pédagogie des Opprimés, Paris, Maspéro, 1973.

FrenCH, J.R.P. et B. RAven, "Les bases du pouvoir social", dans A. Levy (éd.), Psychologie Sociale: Textes Fondamentaux, Tome 1, Paris : Dunod, 1965 : 359-375.

Friedberg, E. et Crozier, M., L'Acteur et le Système, Paris, Éditions du Seuil, 1977.

Fromm, E., The Anatomy of Human Destructiveness, New York, Bantam, 1975.

GalPeR, J., The Politicis of Social Services, Englewood Cliffs (N.J.), Prentice-Hall, 1975.

GalPer, J., Social Work Practice: A Radical Perspective, Englewood Cliffs (N.J.), Prentice-Hall, 1980.

Goldmann, Lucien, La Création Culturelle dans la Société Moderne, Paris, Denoel, 1971.

Gordon, D., Therapeutic Metaphors, Cupertino, (Cal.) Meta Publications, 1978.

GuÉRIN, P.J. et Pendagast, E.G., "Evaluation of Family System and Genogram ", dans Family Therapy, P.J. Guérin (éd.), New York, Gardner Press, 1976: 450-464.

HASHIMI, J.K., "Environmental Modification: Teaching Social Coping Skills", Social Work, vol. 26, no 4, 1981 : 323-326.

Hepworth, D.H. et LarSen, J.H., Direct Social Work Practice, Dorsey Press, Homewood (III.), chapitre 18, "Additive Empathy and Confrontation", 1982 : 397-416.

KeEfE, T., "Empathy Skill and Critical Consciousness ", Social Casework, vol. 61, no $7,1980: 387-393$.

KOtKer, Z., "The Feminine Behavior of Powerless People", Savvy, 1980 : 36-45.

LAMONT, S., "L'Approche Structurelle en Travail Social: Une Action Possible pour Guider une Pratique Transformatrice", Recueil de Textes - Méthodologie de l'Intervention, École de service social, Université de Montréal, 1987-1988 : 2-33.

LASCH, C., Haven in a Heartless World: The Family Besieged, New York, Basic Books, 1979.

LeONARD, P., "A Paradigm for Radical Practice ", dans Bailey, R., et M. Brake (eds) Radical Social Work, London, Edward Arnold, 1975: 46-81.

LEONARD, P., Personality and Ideology: Towards a Materialist Understanding of the Individual, London, MacMillan, 1984.

Lerner, M.P., Clinical and Therapeutic Aspects of the Social Psychology of Freedom and Powerlessness: The Foundations of the Psychogenesis Process, Thèse de Doctorat non publiée, Wright Institute Graduate School, Ann Arbor (Mic.), University Microfilm, 1977. 
LeVIne, H. et Estable, A., Rapports de Force et Maternité : une Critique Féministe de la Théorie et de la Pratique, Cahier publié par le Center for Social Welfare Studies, Ottawa, École de service social, Université Carleton, 1982.

Middleman, R.R. et GoldberG, G., Social Service Delivery: A Structural Approach to Social Work Practice, New York and London, Columbia University Press, 1974.

MISPELBLOM, F., " Pratiques sociohistoriques en Travail Social », Revue Internationale d'Action Communautaire, vol. 8, $\mathrm{n}^{\circ} 48,1982$ : 177-190.

Mispelblom, F., "Pistes pour une pratique silencieuse", Contradictions, n० 29, $1981: 71-100$.

Moreau, M., "A Structural Approach to Social Work Practice ", Revue canadienne des Écoles de service social, vol. 5, no 1, 1979 : 78-94.

MoREAU, M., "L'approche structurelle familiale en service social : le résultat d'un itinéraire critique ", Revue internationale d'Action Communautaire, vol. 7, no 47, 1982: 159-171.

Moreau, M., "Empowerment as an Antidote to Despair in our Welfare/Workfare World: Clinical and Policy, Practice Implications of a Structural Approach to Social Work", article non publié, Université de Montréal, École de service social, Août 1986.

Moreau, M., "Rules Reconstruction ", notes non publiées (1977) sur une méthode adaptée de la Reconstruction Familiale développée par Virginia Satir, à Esalen (Cal.) (1968) et à Bellingham (Wash.), (1973) École de service social, Université Carleton, Ottawa.

Moreau, M., A Structural Approach in Practice, Rapport de recherche non publié subventionné par Santé et Bien-Être Canada, 1988.

Mueller, K. et LeIDIG, M.W., "Women's Anger and Feminist Therapy ", Frontiers, vol. 1, no 3, 1976: 23-30.

Netson-Jones, R., Personal Responsibility Counseling and Therapy: An Integrative Approach, London, Harper and Row, 1984.

PANET-RAYMOND, J., L'enseignement du changement politique dans un contexte politique changeant, présentation au 24e Congrès International de l'Association internationale des Écoles de service social, Vienne, juillet 1988.

PATtI, R.J. et ResNiCK, H., "Changing the Agency from Within ", Social Work, vol. 17, no 4, 1972: 48-57.

Pearson, G., "Making Social Workers. Bad Promises and Good Owens", dans R. Bailey et M. Brake (éds), Radical Social Work, London, Edward Arnold, 1975: 13-45.

PINCUS, A., "Reminiscence in Aging and its Implications for Social Work Practice ", Social Work, 15, no 3, 1970 : 47-53.

RENÉ, L., "Approche structurelle et intervention individuelle: une réflexion à partir d'une pratique en centre d'accueil », travail de Maîtrise non publié, Montréal, École de service social, Université de Montréal, décembre 1987.

RıCH, A., "La Contrainte à l'hétérosexualité et l'existence lesbienne ", Nouvelles Questions Féministes, vol. 1, no 1, 1981: 15-43.

SATIR, V., Peoplemaking, Palo Alto, (col.) Science and Behavior Books, 1972.

ScHon, D.A., The Reflective Practitioner, New York, Basic Books, 1983. 
SCHon, D.A., Educating the Reflective Practitioner, San Francisco, Jossey-Bass, 1987.

Sève, L., Marxisme et théorie de la Personnalité, Paris, Éditions Sociales, 1979.

SHATZ, E., " The Defense of Socially Structured Inequality : A Theoretical Analysis ", Thèse de Doctorat non publiée, Brandeis University, The Florence Heller Graduate School for Advanced Studies in Social Welfare, 1973.

SimONS, R.L., "Strategies for Exercising Influence", Social Work, vol. 27, no 3, 1982: 268-273.

SIMPKIN, M., Trapped Within Welfare : Surviving Social Work, London, MacMillan, 1983.

SPECK, R.V. et RuEvenI, U., "Network Therapy: A Developing Concept ", Family Process, vol. 8, no 2, 1969: 182-191.

Statham, D., Radicals in Social Work, London, Routledge et Kegan Paul, 1978.

Steiner, C., Scripts People Live, New York, Bantam, 1974.

STEPHEN, R. and BLACK, B., Advocacy and Empowerment: Mental Health Care in the Community, Boston, London Routledge and Kegan Paul, 1985.

SUE, G., Wing et SUE, D., "Barriers to Effective Cross-Cultural Counseling", Journal of Counseling Psychology, 1977, vol. 34, no 3 : 420-429.

SzASz, T.S., The Myth of Mental Illness: Foundation of a Theory of Personal Conduct, New York, Paul B. Hoeber, 1961.

TURNER, J.B., "Education for Practice with Minorities ", Social Work, vol. 17, no 3, 1972: 112-118.

Walker, H. et Beaumont, B., Probation Work: Critical Theory and Socialist Practice, Oxford, Basil Blackwell, 1981.

Wickoff, H., Solving Women's Problems, New York, Grove Press, 1977. 